Synthesis of Natural

Products and

Potential Drugs

\section{Synthesis of Alsmaphorazine B}<smiles>NCCc1c[nH]c2ccccc12</smiles>

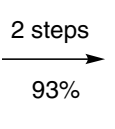<smiles>CC(=O)C1=C(C(C)=O)C2(O)CCC1CN1CCC23c2ccccc2NC13</smiles>

E

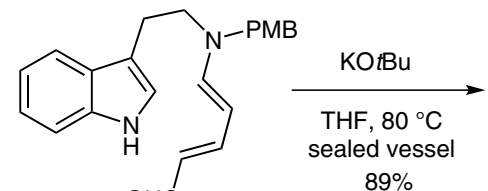

B $\mathrm{OHC}$<smiles>CCCCCN1CCC23c4ccccc4NC2C(C=O)=CCC13</smiles>

C
1. $\mathrm{K}_{2} \mathrm{OsO}_{4} \cdot 2 \mathrm{H}_{2} \mathrm{O}(10 \mathrm{~mol} \%)$ $\mathrm{NMO}$, citric acid

$t-\mathrm{BuOH}-\mathrm{H}_{2} \mathrm{O}(1: 1)$ 2. DMP, $t-\mathrm{BuOH}, \mathrm{CH}_{2} \mathrm{Cl}_{2}$

$68 \%$ Diels-Alder cycloaddition<smiles></smiles>

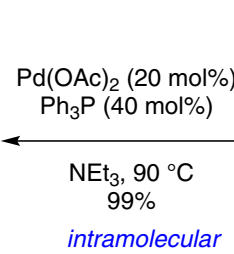

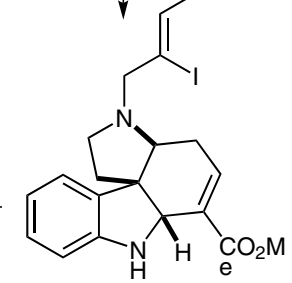

\section{Key words}

alsmaphorazine B

akuammicine

Heck reaction

Cope elimination

1,3-dipolar cycloaddition

isoxazolidine alkaloids<smiles>CC(=O)C1=C(C(C)=O)C2CCC1CN1CCC2(C)C1</smiles>

Alstolucine $\mathbf{F}$ (33\%)

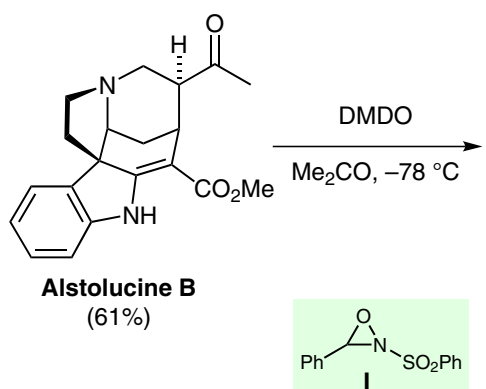

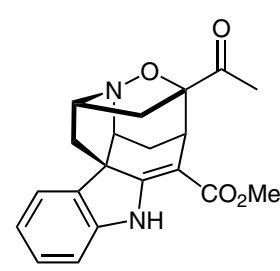<smiles>COC(=O)C1=C2Nc3ccccc3C23CC[N+]([O-])(CC3)C[C@H]1C(C)=O</smiles>

F

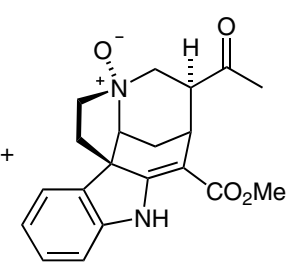

Alstolucine C (61\%)
LiHMDS, THF then 1 $-78{ }^{\circ} \mathrm{C}$ to $0{ }^{\circ} \mathrm{C}$ $82 \%$

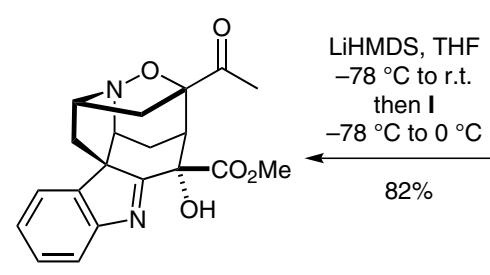

Alsmaphorazine B

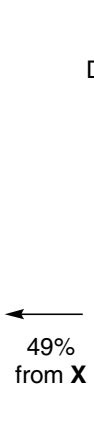
$49 \%$
from $X$ $\mathrm{O}_{2}, 80^{\circ} \mathrm{C} \sqrt{ }$ Cope elimination
Significance: Alsmaphorazine B is an indole alkaloid endowed with an isoaxzolidine embedded in the hexacyclic skeleton. Biosynthetically, this natural product is believed to originate from the related alkaloid akuammicine. Hong and Vanderwal present a short synthetic route employing an oxidation/Cope elimination/cycloaddition sequence to enable the transformation of akuammicine into alsmaphorazine B.
Comment: A previously employed intramolecular Heck reaction provided high-yielding access to acuammicine from $\mathbf{D}$. Oxidation followed by $\mathrm{Sml}_{2}$ mediated deoxygenation gave alstolucines $\mathrm{B}$ and F. Oxidation with DMDO followed by base induced Cope elimination yielded hydroxylamine $\mathbf{G}$, which upon oxidation to the nitrone underwent 1,3-dipolar cycloaddition to $\mathbf{H}$. Finally, $\alpha$-hydroxylation furnished alsmaphorazine B in 15 steps and 11\% overall yield.

SYNFACTS Contributors: Erick M. Carreira, Christian Ebner 